\title{
A Fluorescence Microscopy Assay for Assessing Beam Damage to Nanoparticle Capping Ligands During Liquid Cell Electron Microscopy
}

\author{
Thilini U. Dissanayake, ${ }^{1}$ Mei Wang, ${ }^{1}$ Taylor J. Woeh ${ }^{1 *}$ \\ 1. Department of Chemical and Biomolecular Engineering, University of Maryland, College Park, MD, \\ USA. \\ *Corresponding author: tjwoehl@umd.edu
}

Liquid cell electron microscopy is commonly used to characterize the dynamics of nanoparticles (NPs) in a liquid medium, often during nanoparticle self-assembly. Many studies have been done in this area to investigate the self-assembly of NPs functionalized with different capping ligands such as small molecules, polymers and DNA $[1,2,3]$. Critically, the specific chemistry of the organic capping ligands on NPs determines their interactions during self-assembly and the morphology of the final self-assembled structure. It is well known that organic materials in liquid undergo oxidative attack from oxidizing radicals and molecules created by the electron beam, including hydroxyl radicals and hydrogen peroxide [4]. However, the effect of electron beam irradiation on the surface functional groups on NPs has not been systematically investigated, which has prohibited discovery of acceptable dose limits for imaging NPs during self-assembly with minimal beam induced changes to their surface chemistry.

In biology, fluorescent molecules are commonly used to specifically label cellular structures such as cells and tissues, to study genetic materials, or to label certain proteins in an intracellular environment. Fluorescent molecules are bound to specific targets using chemical conjugation to form covalent bonds between the fluorophore and functional group of interest on a molecule or protein. Ideally, fluorescent tags are highly specific to certain functional groups, including amines, carboxylic acids, alcohols, and thiols [5]. The position of fluorescent tags can then be localized using fluorescence microscopy. In this work, we borrow this concept to label functional groups on NPs that have been exposed to the electron beam in a liquid cell.

Overall, we have developed a fluorescence assay to measure electron beam induced modification of nanoparticle capping ligands during liquid cell scanning transmission electron microscopy (LC-STEM) imaging. First to test whether the assay is specific to a certain type of capping ligand, we performed control experiments where the amine capping ligands on silver nanoparticles were removed with a strong oxygen plasma. Silver NPs $(100 \mathrm{~nm})$ functionalized with amine groups were deposited onto two silicone chips and capping ligands of NPs on one chip were removed using oxygen plasma. NPs were subsequently fluorescently labeled with an amine reactive dye conjugation, carboxy-flourescein, using standard amide coupling chemistry. Fluorescence microscopy showed no fluorescence for NPs that were plasma cleaned and bright green fluorescence for NPs with intact amine surface ligands (Figure 1), demonstrating specificity of this assay to the target capping ligand. We applied this assay to a LC-STEM sample, consisting of $100 \mathrm{~nm}$ amine functionalized silver NPs deposited on the silicon nitride membranes of the liquid cell. The cell was filled with DI water and NPs in different areas of the liquid cell were imaged for different amounts of time and different electron beam currents. The silver NPs were exposed to the electron beam for 1, 5, and 10 minutes with beam currents of 31 and $207 \mathrm{pA}$ at a magnification of $20 \mathrm{kx}$. The sample was disassembled after the TEM imaging and the NPs were labeled with carboxy-fluorescein as described above. Figure 2 shows fluorescence microscopy images of a few areas that were imaged during LC-STEM. The images show no significant decrease in the fluorescence intensity of the NPs (small green spots), but they do show fluorescent squares in the region imaged with the STEM beam. The 
intensity of the green squares increased with imaging time and total dose. We believe these green squares are a result of electron beam induced cleavage and redeposition of amine capping ligands on the silicon nitride membrane. The assay can be easily modified to test effects of beam damage on other capping ligands by using fluorescent molecules with specificity to other functional groups. This approach should enable establishing LC-STEM imaging conditions under which the capping ligands on nanoparticles are preserved and not significantly damaged during in situ self-assembly experiments [6].

\section{References:}

[1] Woehl, T.J. and Prozorov. T., J. Phys. Chem. C, 119(36) (2015), p. 21261.

[2] Luo, B., Smith, J.W., Ou, Z., Chen, Q., Acc. Chem. Res., 50(5) (2017), p. 1125.

[3] Jungjohann, K. L. et al., Micron, 119 (2019), p. 54.

[4] Woehl, T.J., and Abellan, P., J. Microscopy, 265(2) (2017), p. 135.

[5] Sanderson, M. J., Smith, I., Parker, I., Bootman, M. D. (2014). Fluorescence microscopy. Cold Spring Harbor protocols, 2014(10)

[6] We acknowledge partial funding from the UMD-UMB strategic partnership seed grant.
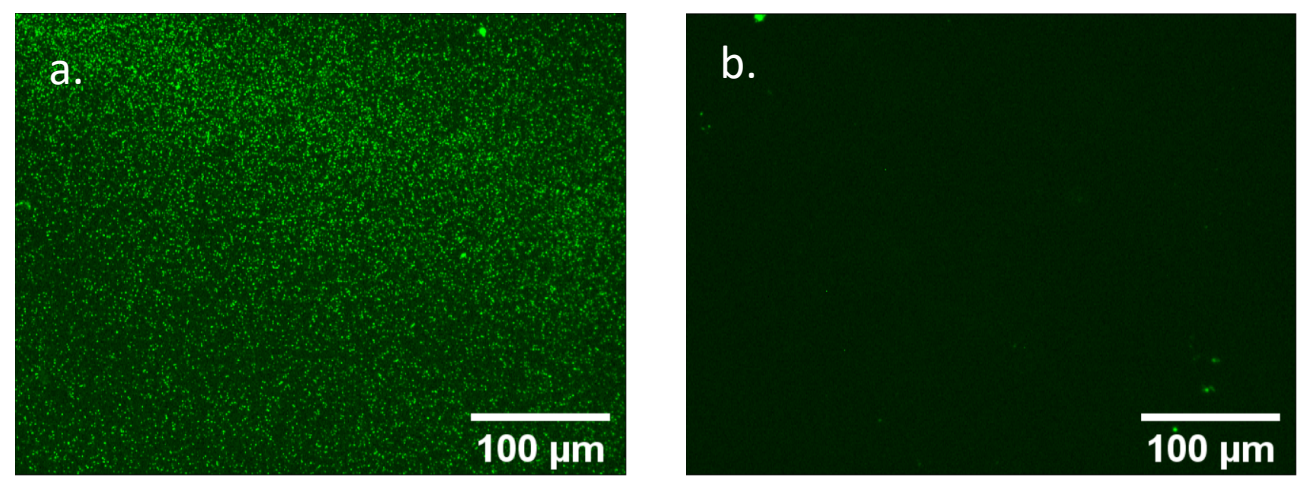

Figure 1. Fluorescence microscopy of fluorescein-labeled amine functionalized $100 \mathrm{~nm}$ silver NPs. a) NPs with intact amine groups. b) NPs with amine groups removed by oxygen plasma.
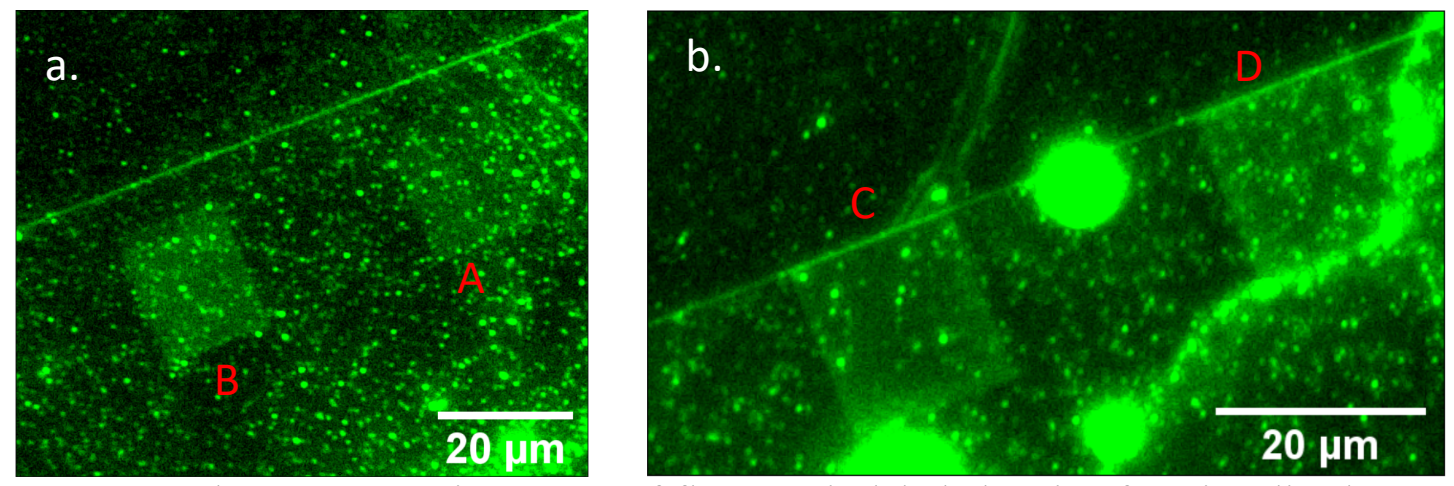

Figure 2. Fluorescence microscopy of fluorescein labeled amine functionalized $100 \mathrm{~nm}$ silver NPs after LC-STEM imaging. a) The beam current was $31 \mathrm{pA}$, magnification was $20 \mathrm{kx}$, giving a dose rate of 0.054 MGy/s (A. Total dose: 16.3 MGy B. Total dose: 32.7 MGy). b) The beam current was 207 pA, magnification was $20 \mathrm{kx}$, giving a dose rate of $0.363 \mathrm{MGy} / \mathrm{s}$ (C. Total dose: 109.0 MGy D. Total dose: 218.0 MGy). 